\title{
The contribution of maternal psychological functioning to infant length of stay in the Neonatal Intensive Care Unit
}

This article was published in the following Dove Press journal:

International Journal of Women's Health

27 June 2016

Number of times this article has been viewed

\author{
Amanda S Cherry' \\ Melissa R Mignogna' \\ Angela Roddenberry Vaz' \\ Carla Hetherington ${ }^{2}$ \\ Mary Anne McCaffree ${ }^{2}$ \\ Michael P Anderson ${ }^{3}$ \\ Stephen R Gillaspy'
}

'Section of General and Community Pediatrics, Department of Pediatrics, University of Oklahoma Health Sciences Center, Oklahoma City, OK, ${ }^{2}$ Neonatal Perinatal Medicine, Department of Pediatrics, University of Oklahoma, College of Medicine, Oklahoma City, OK, ${ }^{3}$ Department of Biostatistics and Epidemiology, University of Oklahoma Health Sciences Center, College of Public Health, Oklahoma City, OK, USA
Correspondence: Amanda S Cherry Section of General and Community Pediatrics, Department of Pediatrics, University of Oklahoma Health Sciences Center, 1200 Children's Ave Suite 12400, Oklahoma City, OK 73104, USA

Tel + I 405 27| 4407

Fax + I 405 27। 8709

Email amanda-cherry@ouhsc.edu
Objective: Assess maternal psychological functioning within the Neonatal Intensive Care Unit (NICU) and its contribution to neonate length of stay (LOS) in the NICU.

Study design: Mothers of infants admitted to the NICU $(n=111)$ were assessed regarding postpartum depression, postpartum social support, postpartum NICU stress, and maternal anxiety at 2 weeks postpartum. Illness severity was assessed with the Clinical Risk Index for Babies (CRIB).

Results: Postpartum depression was not significantly correlated with LOS, but was significantly correlated with trait anxiety ( $r=0.620)$, which was significantly correlated with LOS $(r=0.227)$. Among mothers with previous mental health history, substance abuse history and CRIB score were the best predictors of LOS. For mothers without a prior mental health issues, delivery type, stress associated with infant appearance, and CRIB scores were the best predictors of LOS. In this group, LOS was found to increase on average by 7.06 days per one unit increase in stress associated with infant appearance among mothers with the same delivery type and CRIB score.

Conclusion: Significant correlations of trait anxiety, stress associated with infant appearance, and parental role with LOS support the tenet that postpartum psychological functioning can be associated with NICU LOS.

Keywords: NICU, postpartum depression, postpartum anxiety, parental stress, CRIB

\section{Introduction}

The Neonatal Intensive Care Unit (NICU) experience can invoke significant environmental and psychological distress. ${ }^{1-4}$ With more than 480,000 premature infants born every year in the USA, we continue to see an increase in premature births, ${ }^{5}$ and utilization of special care nursery settings such as the NICU. An increasing body of literature suggests that postpartum depression (PPD) and several related psychosocial variables, such as anxiety, social support, and stress associated with the NICU environment, are significantly related to both infant and parental outcomes. ${ }^{6}$

Parents of NICU infants commonly experience high levels of stress and feelings of helplessness; which often reflect a lack of knowledge of how to parent, interact, and care for their neonates during the NICU stay. ${ }^{7-9}$ More specifically, mothers of premature and low-birth-weight infants have been reported to experience more considerable levels of psychological distress, and to be at greater risk for relationship difficulties, family stress, and financial stress, when compared with mothers of term infants. ${ }^{10}$ Much of the psychological distress mothers of NICU infants experience is related to concerns about the long-term outcomes and/or survival of the infant; 
having an altered parental experience; being separated from the infant; feelings of limitations about their ability to care for the infant; and feelings of helplessness. ${ }^{11}$ Complex medical terminology, separation from their infants, and anxiety about long-term consequences of the preterm birth lend mothers of preterm infants to be more prone to experiencing anxiety and depression. ${ }^{12}$ Additionally, mothers of neonates in the NICU frequently experience situations that are seemingly out of their control, which can result in more difficulty developing perceptions of mastery/control, placing these mothers at higher risk for distress, anxiety, and depression. ${ }^{13}$

PPD is commonly considered the most frequent and significant mental health complication of childbirth. Approximately $15 \%$ of all mothers experience symptoms meeting full diagnostic criteria for PPD, while as many as 50\%-80\% experience postpartum symptoms such as fatigue and mood swings. ${ }^{14}$ PPD rates have been found to be significantly higher in mothers of preterm infants than mothers who deliver full-term infants. ${ }^{15-17}$ More specifically, compared with the general population, mothers of preterm infants are $40 \%$ more likely to develop PDD. ${ }^{4}$ Among the highest PPD rates are mothers of neonates in the NICU, ${ }^{18}$ as it has been reported that $28 \%-70 \%$ of mothers of infants in NICUs experience PPD. ${ }^{19}$ One of the most significant contributors of depressive symptoms for mothers of neonates in the NICU has been the perceived loss in carrying out maternal roles. ${ }^{2}$

It has also been reported that in comparison to control mothers, NICU mothers also experience significantly higher rates of anxiety. ${ }^{10}$ Duration of anxiety following the birth of a preterm infant can persist for more than a year following the birth. ${ }^{20}$ The anxiety reported by mothers of preterm infants has been suggested to be related to NICU stress, but not general distress. ${ }^{21}$ As mentioned previously, the NICU environment can lend parents to perceptions of lower mastery of their situation and thus they are more prone to distress, anxiety, and depression. ${ }^{1}$ The level of psychological distress experienced by mothers of neonates is concerning due to the impact on developing parent-child relationships. Mothers are less likely to interact with their neonates in ways that promote cognitive development when they are experiencing high levels of distress. ${ }^{22}$ Additional effects of high maternal distress and anxiety include less maternal warmth and flexibility, ${ }^{23}$ less sensitive and more controlling maternal behavior, ${ }^{24}$ and feeding and sleep disturbances in the infant. ${ }^{25}$ These are particularly important considerations for NICU infants due to their tendencies to require greater parental support in fostering cognitive and social development given the health complications that often accompany premature birth. ${ }^{26}$
Universal screening for PPD and anxiety are implicated for many reasons, including the high frequency of PPD occurrence in this population, the high potential for longterm implications on their infants, ${ }^{19}$ and to determine which families are at greatest risk for parent-infant relationship problems. ${ }^{20}$ Additionally, universal screening should also include screening for previous mental health concern, as prior mental health problems have been cited as a significant risk factor for the development of PPD. ${ }^{27-29}$ The utilization of universal screening offers an opportunity to identify women with preexisting, undiagnosed, and new-onset symptoms. Regardless the onset of psychosocial symptoms, the risk to the mothers' and the infants' health and development is present, and preterm infants are particularly at risk due to the additional risks for growth and developmental problems. ${ }^{19}$

While it has been clearly established that postpartum anxiety, depression, and distress have significant implications and are more likely to be present in mothers of neonates in the NICU, very little is known about the contribution of PPD, anxiety, and related psychosocial variables to neonate length of stay (LOS) in the NICU. Further, one such example of related psychosocial variables is prior mental health problems. Previous studies have indicated that a history of mental health problems can increase the risk for PPD; however, very little is known about the contribution of previous mental health history to LOS in the NICU. One randomized intervention study revealed that mothers who received an educational-behavioral intervention reported significantly less stress in the NICU and less depression and anxiety at 2 months' corrected infant age than did comparison mothers. ${ }^{6}$ Furthermore, infants of parents in the educational-behavioral intervention had a 3.8-day shorter NICU LOS and a 3.9-day shorter total hospital LOS than did comparison infants. ${ }^{6}$ These results suggest that PPD and related psychosocial variables are related to parental functioning and therefore NICU LOS. A review of the literature reveals no empirical investigation of the specific relationship between PPD, postpartum anxiety, and associated psychosocial variables with NICU LOS.

The long-term goal of this project was to improve the health and quality of life of infants seen in the NICU and the mothers of these neonates. The objectives which were a step toward attainment of this long-term goal were 1) to assess PPD and related psychosocial variables in mothers of neonates admitted to the NICU and 2) assess the contribution of PPD and related psychosocial variables to neonate LOS in the NICU. Further, the goal was to assess the contribution of PPD and psychosocial variables to neonate LOS above and 
beyond the influence of illness severity. Specifically, PPD, postpartum social support, postpartum NICU stress, and maternal anxiety were assessed. The central tenet was that poor maternal psychological functioning would be associated with increased neonate LOS in the NICU.

It was hypothesized that higher levels of PPD, higher levels of maternal anxiety, and higher levels of stress related to the NICU environment would be positively correlated with NICU LOS, and higher levels of NICU social support would be negatively correlated with NICU LOS. It was further hypothesized that these psychosocial variables would significantly contribute to NICU LOS, after statistically controlling for the effects of demographic variables and neonate illness severity.

\section{Methods}

\section{Sample}

Mothers of neonates admitted to the NICU were recruited from a NICU in the south central region of the USA. The NICU from which participants were recruited has $\sim 1,200$ admissions yearly, with an average LOS of 3 weeks, and a mortality rate of $10 \%-15 \%$. Premature infants as well as infants born at or near term are admitted and between $30 \%$ and $40 \%$ of admissions are transfers from other facilities. Mothers were excluded from participation in the study if they read below the eighth grade level; did not speak English; or had infants with congenital anomalies, surgery requirements, significant cardiac abnormalities, or chromosomal defects.

Recruitment of mothers of infants in the NICU began in February 2008 and ended in February 2009. During that time frame, a total of 1,123 infants were admitted to the NICU, of which 463 infants were hospitalized in the NICU for 14 or greater days postpartum. Of these 463 infants, 279 mothers were eligible to participate in the study, and ultimately 111 ( $40 \%$ of eligible) participated in the study. Mothers of NICU infants with congenital anomalies, who required surgery, who had significant cardiac abnormalities, or who had chromosomal defects were excluded from participating. Additionally, mothers who read below an eighth grade level and who did not speak English were excluded. Thus, a large number of mothers with infants hospitalized for greater for 14 days were ineligible to participate given these exclusion criteria. Of those who were eligible and declined participation, the most common reason was not wanting to take the time to participate (Figure 1).

\section{Instruments}

\section{Demographics}

Participants completed a brief demographics questionnaire designed specifically for this study that assessed demographic variables, child birth history, mental health, and substance abuse history.

\section{Postpartum depression}

The PPD Screening Scale (PDSS) ${ }^{30}$ is a 35 -item self-report Likert scale used to assess symptoms of PPD. The PDSS has established validity and good internal consistency, $0.83-0.94 .{ }^{30}$ Total PDSS score was used in the analyses to assess maternal report of PPD symptoms. Within this study, internal consistency (coefficient alpha) was 0.953 for the total PDSS score. Although the PDSS has not been normed in the NICU population, it has been used by previous studies to assess maternal depression for mothers of NICU infants. ${ }^{6}$ McCabe et $\mathrm{al}^{18}$ found the psychometric properties of the PDSS scores to be well supported with excellent estimates of internal consistency of total PDSS scores and adequate to good for subscale scores, with a NICU population.

\section{Maternal anxiety}

The State-Trait Anxiety Inventory (STAI) ${ }^{31}$ is a 20 -item selfreport measure used to assess anxiety. The STAI has been used to assess maternal anxiety in the NICU setting, with

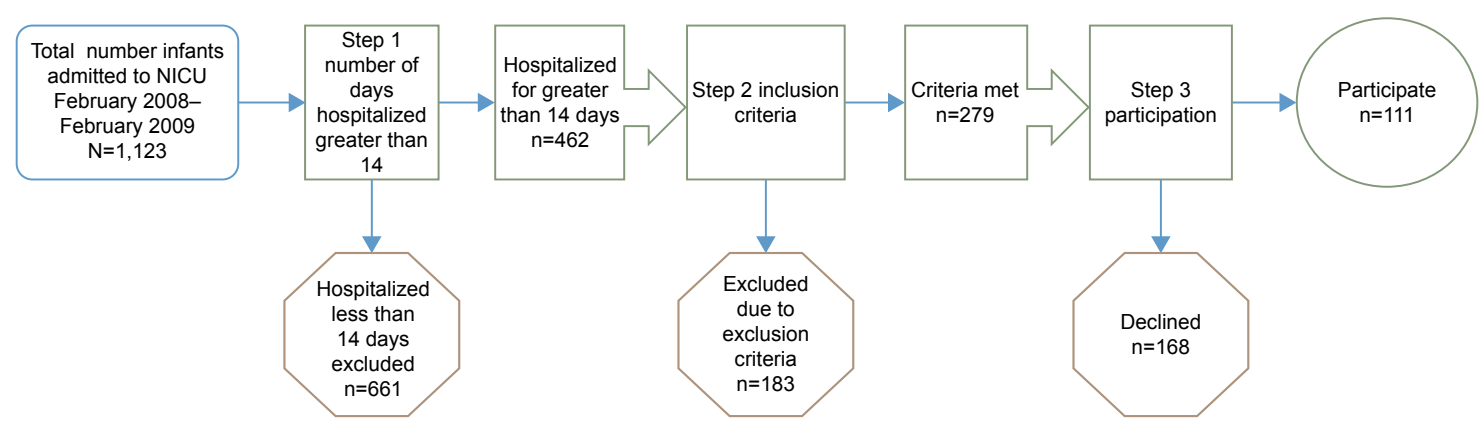

Figure I Maternal participation process.

Abbreviation: NICU, Neonatal Intensive Care Unit. 
internal consistency estimates ranging from 0.83 to $0.95 .^{6}$ Internal consistency within this sample was consistent with Cronbach's alpha $=0.891$. Total STAI score was used in the analyses to assess maternal anxiety.

\section{Postpartum NICU stress}

The Parental Stressor Scale-NICU (PSS-NICU) ${ }^{32}$ is a 46-item self-report measure used to assess parental perception of stressors arising from the physical and psychosocial environment of the NICU. Recent use of the PSS-NICU demonstrated internal consistency estimates of $0.94-0.96 .{ }^{6}$ For this sample, internal consistency was 0.943 and consistent with previous estimates. Total PSS-NICU score was used in the analyses to assess maternal level of stress. Available metrics allow scoring for stress occurrence levels, overall stress levels, and number of stressors experienced. Additionally, the three subscales: Parental Role Alterations, Sights and Sounds of the Unit, and Infant Behavior and Appearance were used to measure stress associated with alternations in the parental role, environmental stress associated with the unit, and the stress associated with the appearance of the infant.

\section{Postpartum social support}

The Postpartum Support Questionnaire (PSQ) ${ }^{33}$ is a 34-item self-report measure used to assess perceived support specific to the postpartum period. The PSQ was designed to assess both the importance of support and support expected or received and has established reliability and validity. ${ }^{33}$ Total PSQ score was used in the analyses to assess level of perceived social support. Internal consistency for the total scale was 0.959 within this sample.

\section{Illness severity}

The Clinical Risk Index for Babies (CRIB) ${ }^{34}$ is a wellestablished measure used to assess neonates' severity of illness. The CRIB was recently used to assess illness severity when investigating the relationship between NICU LOS and parental mental health outcomes. ${ }^{6}$

\section{Length of stay}

Infant NICU LOS is defined as the number of days of hospitalization in the NICU.

\section{Procedure}

This study was approved by the University of Oklahoma Health Sciences Center Institutional Review Board prior to recruitment. Participant eligibility was identified by the project coordinator upon admission to the NICU. Following the second week postpartum, the study coordinator or research assistant, who was trained in the process of informed consent and Health Insurance Portability and Accountability Act research guidelines, approached eligible participants. Mothers were given the measures to complete, following discussion of research privacy forms and consent obtainment. Completion of screening measures was considered minimal risk to participants. However, critical items on screening measures were immediately reviewed by the research assistant, and immediate psychological consultation was made available for significant depressive symptoms or suicidality reported on the PDSS. Additionally, all participants were provided with information regarding community resources for psychological services. The project coordinator accessed the child's medical record and completed the infant's illness severity (CRIB score).

\section{Data analysis}

The data analysis involved multiple steps. First, descriptive statistics regarding the characteristics of the sample were analyzed. Coefficient alpha was calculated for each of the total scales and subscales. Additionally, descriptive statistics of the reporting trends of the study's sample were completed for each instrument administered. Second, bivariate results were analyzed in order to inform of confounding variables. Associations between demographic variables (age, ethnicity, marital status, work status, level of income, child birth history, mental health, and substance abuse history) and the predictor variable (illness severity), as well as associations with each demographic variable and dependent variable, LOS, were examined with zero-order correlations. Demographic variables found to be significantly correlated with LOS and illness severity were controlled for in primary analysis. Then finally, multivariate models were completed for those without mental health history and those with mental health history regarding LOS. Because LOS was not normally distributed, iteratively reweighted least squares (robust) regression was used to test the independent effect of the covariates on LOS. This estimation procedure minimizes the effect of outliers in the dependent variable LOS to provide reliable estimates of the model parameters. Variables considered for modeling included mental health history (ie, depression, anxiety, and substance abuse), delivery type, stress related to the infant's appearance, alteration of the parental roles, birth weight, and gestational age.

\section{Results \\ Descriptive statistics}

The mean age of the sample was 29.35 years (standard deviation $[\mathrm{SD}]=5.48$, range: $18-40$ ). Several reported previous 
mental health concerns, including depression (25\%), anxiety $(23 \%)$, and substance abuse (7\%). Additional demographic characteristics for race, marital status, education, mental health history, and insurance status are provided in Table 1. Many of the women reported that this was their first pregnancy $(39.1 \%)$, and the majority reported that the pregnancy was not planned (63.1\%). Descriptive characteristics of the participants' infants are given in Table 2.

Based on responses to the Postpartum Screening Scale, most of the women $(52.3 \%)$ received a positive screening for major PPD, indicating the need for a referral to a mental health professional for further evaluation. Next, 29.7\% received a score significant for symptoms of PPD, indicating the possible need for a referral to a mental health professional. These mothers are reporting significant symptoms, but do not reach the threshold of clinical significance; however, are at high risk and reasonable to consider a referral to a mental health professional. The remaining $18.0 \%$ received a score indicative of normal adjustment after the birth of a baby. The mean score for trait anxiety on the STAI was $49 \%$ ( $\mathrm{SD}=9.66)$, which was significantly higher than the published norms for working adult females as reported in the manual ( mean $=34.79, \mathrm{SD}=9.22$ ).

Parental stress, as measured by the PSS-NICU, resulted in participant responses with mean stress ratings as follows: Parental Role 3.45, Infant Appearance 2.92, and Sight and

Table I Demographic information for participants

\begin{tabular}{|c|c|c|}
\hline Variable & n (total = I II) & $\%$ of sample \\
\hline \multicolumn{3}{|l|}{ Race } \\
\hline Caucasian & 68 & 61.3 \\
\hline African American & 20 & 18.0 \\
\hline Native American & 12 & 10.8 \\
\hline Hispanic & 6 & 5.4 \\
\hline Other & 5 & 4.5 \\
\hline \multicolumn{3}{|l|}{ Marital status } \\
\hline Married & 48 & 43.2 \\
\hline Single parent & 25 & 22.5 \\
\hline Partnered & 31 & 27.5 \\
\hline Divorced & 4 & 3.6 \\
\hline Separated & 3 & 2.7 \\
\hline \multicolumn{3}{|l|}{ Education } \\
\hline Some college or higher & 55 & 49.5 \\
\hline High school diploma & 35 & 31.5 \\
\hline Some high school & 20 & 18.0 \\
\hline \multicolumn{3}{|l|}{ Prior mental health history } \\
\hline Depression & 28 & 25.2 \\
\hline Anxiety & 25 & 22.5 \\
\hline Substance use disorder & 8 & 7.2 \\
\hline \multicolumn{3}{|l|}{ Insurance } \\
\hline Medicaid & 89 & 77.5 \\
\hline Private & 19 & 17.1 \\
\hline Both & 3 & 2.7 \\
\hline
\end{tabular}

Table 2 Sample infant characteristics

\begin{tabular}{lll}
\hline Covariates & n & Median (25th\%-75th\%) \\
\hline Gestational age & III & $30.0(27.0,33.0)$ \\
Birth weight & 106 & $1.75(1.08,3.10)$ \\
Length of stay & III & $39.0(23.0,70.0)$ \\
Multiple gestation: "No" count (\%) & & $90(84.91)$ \\
\hline
\end{tabular}

Sound 2.64 (on a 1-5 Likert scale). Overall, participants rated the stress related to perceived loss of parental role as the most stressful experience related to being in the NICU. Based on responses to the PSQ, there were no significant differences between reported importance of material, information, emotional, or competence support and reported support received. However, the majority of the social support subscales were significantly correlated with each other.

\section{Bivariate relationships}

Regarding relationships with NICU LOS, total PPD scores were not significantly correlated with NICU LOS. However, total PPD scores were significantly correlated with prior depression $(r=-0.230)$, prior drug/ alcohol treatment $(r=-0.215)$, support of material importance $(r=0.225)$, stress related to infant appearance $(r=0.378)$, stress related to different parental roles $(r=0.490)$, stress related to the sights and sounds of the NICU $(r=0.341)$, and STAI total $(r=0.620)$. NICU LOS was significantly correlated with the total score for trait anxiety on the STAI ( $r=0.227)$. NICU LOS was also significantly correlated with stresses associated with the NICU environment including infant appearance $(r=0.321)$ and changes in parental role $(r=0.201)$. No social support subscales were significantly correlated with NICU LOS.

\section{Multivariable models}

Because LOS was not normally distributed, iteratively reweighted least squares (robust) regression was used to test the independent effect of the covariates on LOS for both groups (previous mental health issues/no previous mental health issues), while controlling for the effect of illness severity. Information regarding characteristics of the participants' infants by group is given in Table 3 .

For mothers with previous mental health issues, substance abuse and CRIB score were the best predictors of LOS. When taking illness severity into account, for mothers with children having the same CRIB score, those who do not have a history of substance abuse will, on average, have an increased LOS of 24.48 days. Likewise, for mothers with the same substance abuse history, LOS will increase, on average, 6.63 days for every one unit increase in CRIB score (Table 4). 
Table 3 Infant characteristics by group

\begin{tabular}{|c|c|c|c|c|}
\hline \multirow[t]{3}{*}{ Covariates } & \multicolumn{4}{|c|}{ Any previous mental health issue? } \\
\hline & \multicolumn{2}{|c|}{ No } & \multicolumn{2}{|c|}{ Yes } \\
\hline & $\mathbf{n}$ & Median (25th\%-75th\%) & $\mathbf{n}$ & Median (25th\%-75th\%) \\
\hline Gestational age & 67 & $30.0(27.0,32.0)$ & 44 & $31.5(27.0,33.0)$ \\
\hline Birth weight & 65 & $1.59(1.06,3.11)$ & 41 & $1.77(1.12,2.94)$ \\
\hline Length of stay & 67 & $45.0(25.0,71.0)$ & 44 & $34.0(19.5,68.5)$ \\
\hline Multiple gestation: “No” count (\%) & & $50(78.13 \%)$ & & 40 (95.24\%) \\
\hline
\end{tabular}

For the group with no previous mental health issues, delivery type, infant appearance, and CRIB score were the best predictors of LOS. Within the group of mothers with no previous mental health issues, vaginal deliveries lead to a decrease in the LOS of 13.70 days on average among mothers with the same infant appearance and CRIB score. Likewise, within this group LOS increases on average by 6.87 days for every one unit increase in infant appearance among mothers of the same delivery type and CRIB score. Also, LOS increases on average by 3.96 days for every one unit increase in CRIB score among mothers of the same delivery type and infant appearance (Table 4).

While controlling for CRIB scores, this study found a good predictive model of LOS for those with previous mental health included the following variables: birth weight, prior anxiety, and substance abuse. This resulted in history of substance abuse $(P=0.0034)$ and prior anxiety $(P=0.0169)$ as predictors of LOS. A good predictive model of LOS for those without previous mental health issues included the following variables: birth weight, delivery type, infant appearance, and STAI score, while controlling for CRIB. This resulted in delivery type $(P=0.0023)$ as predictive of LOS for mothers with no prior history of mental health issues (Table 5).

\section{Discussion}

The majority of participants had a positive screening for PPD, anxiety, and stress related to the NICU environment. PPD and maternal anxiety are significant problems as they affect how a woman cares for herself as well as her infant. ${ }^{19}$
The results of the current study suggest that PPD alone does not predict NICU LOS; however, previous studies have suggested that NICU LOS is a significant risk factor for PPD and psychological distress..$^{29,35}$

Identifying and referring women with PPD for mental health services within the NICU environment is implicated given the frequency of PPD occurrence in this population as well as the high potential for long-term implications on their infants. ${ }^{19}$ Screening of maternal anxiety and the social risk factors of anxiety in the NICU population is imperative to determine which families are at greatest risk for parentinfant relationship problems. ${ }^{20}$ Women with neonates in the NICU are at a high risk for depression and anxiety, failing to identify and treat these women will likely result in extending the duration of symptoms. ${ }^{19}$ Previous reports have suggested that $46 \%$ of women continue to have symptoms 1 year after the birth of their children who experienced depressive symptoms postpartum. ${ }^{19}$

Although PPD was not significantly correlated with NICU LOS, it was significantly correlated with trait anxiety, which was significantly correlated with LOS. Due to a highly significant correlation between PPD scores and trait anxiety scores, it is possible that they are measuring the same or similar phenomena in this population. The significant correlations of trait anxiety as well as the stress of infant appearance, type of delivery, substance use, and stress of parental role with LOS in the NICU support the tenet that postpartum psychological functioning is related to LOS for infants in the NICU.

Table 4 Regression parameters for mothers with previous mental health issues and no previous mental health issues

\begin{tabular}{llll}
\hline Mental health history & Variable & Regression parameter $\mathbf{( 9 5 \% \mathbf { C I } )}$ & $\mathbf{P}$-value \\
\hline Previous mental health issues & Intercept & 10.75 & $\mathrm{NA}$ \\
& Substance abuse & $24.48(8.06,40.9 \mathrm{I})$ & 0.0035 \\
& CRIB score & $6.63(4.66,8.60)$ & $<0.000$ I \\
No previous mental health issues & Intercept & 30.77 & $\mathrm{NA}$ \\
& Delivery type & $-13.70(-24.02,-3.37)$ & 0.0093 \\
& Infant appearance & $6.87(1.87,11.87)$ & $0.007 \mathrm{I}$ \\
& CRIB score & $3.96(2.65,5.27)$ & $<0.000$ I \\
\hline
\end{tabular}

Abbreviations: $\mathrm{Cl}$, confidence interval; CRIB, Clinical Risk Index for Babies; NA, not available. 
Table 5 Analyses stratified by previous mental health issues

\begin{tabular}{|c|c|c|c|c|}
\hline \multirow[b]{2}{*}{ Spearman correlation with LOS } & \multicolumn{2}{|c|}{ Previous mental health issues } & \multicolumn{2}{|c|}{ No previous mental health issues } \\
\hline & Birth weight & $r=-0.770$ & Birth weight & $r=-0.697$ \\
\hline & CRIB score & $r=0.666$ & CRIB score & $r=0.708$ \\
\hline & Gestational age & $r=-0.880$ & Gestational age & $r=-0.870$ \\
\hline & Infant appearance & $r=0.299$ & Infant appearance & $r=0.409$ \\
\hline & & & PDSS ELB & $r=0.266$ \\
\hline & & & PDSS total & $r=0.243$ \\
\hline & & & Parental role & $r=0.318$ \\
\hline & & & STAI & $r=0.381$ \\
\hline Spearman correlation with LOS, controlling for CRIB & Birth weight & $r=-0.617$ & Birth weight & $r=-0.460$ \\
\hline \multirow[t]{3}{*}{ score } & Gestational age & $r=-0.789$ & Gestational age & $r=-0.752$ \\
\hline & & & Infant appearance & $r=0.271$ \\
\hline & & & STAI & $r=0.267$ \\
\hline \multirow[t]{2}{*}{ Categorical predictors of LOS (Wilcoxon test) } & Prior anxiety & $P=0.0034$ & Delivery type PPD severe & $P=0.0007$ \\
\hline & & & & $P=0.0295$ \\
\hline Categorical predictors of LOS, controlling for CRIB score & Substance abuse & $P=0.0034$ & Delivery type & $P=0.0023$ \\
\hline (variables tested individually, not together, using ANOVA) & Prior anxiety & $P=0.0169$ & & \\
\hline
\end{tabular}

Note: Only statistically significant analysis results are shown.

Abbreviations: ANOVA, analysis of variance; CRIB, Clinical Risk Index for Babies; LOS, length of stay; PPD, postpartum depression; STAI, State-Trait Anxiety Inventory; PDSS, postpartum depression screening scale; ELB, emotional lability.

\section{Mental health history and LOS}

The results of the current study also suggest that LOS may be impacted by different factors for those mothers with a history of prior mental health issues (eg, substance abuse, illness severity) and those without prior mental health history (eg, delivery type, stress related to appearance, illness severity). Thus, it is imperative to not only screen mothers of NICU infants regarding current psychological functioning, but also previous mental health history in order to develop appropriate interventions targeted at improving parent and child adjustment. Previous mental health concerns have been repeatedly cited in the literature as a risk factor for $\mathrm{PPD}^{27-29}$ and the results of this study suggest that LOS is impacted differently by maternal functioning of a mother with previous mental health concerns than that of a mother without previous mental health concerns.

\section{Implications for mothers with mental health history}

As previously mentioned, prior mental health problems have been cited as a significant risk factor for the development of PPD. ${ }^{27-29}$ Of those mothers reporting a history of previous mental health concerns within the current study, prior substance abuse $(P=0.0035)$ was the only significant categorical predictors of LOS while controlling for CRIB scores. Therefore, while previous studies have suggested that previous mental health concerns increase the risk of PPD, the results of this study suggest that of those mothers with previous mental health concerns, substance abuse was predictive of LOS.

Further, one surprising result of this study indicated a negative relationship between a history of substance abuse and LOS. This result may suggest that mothers with a history of previous mental health concerns without a history of substance abuse are likely to have longer LOS, than those mothers with a history of substance abuse only. With an increase of LOS, on average, 24.48 days for mothers with previous mental health concerns without a history of substance abuse, there is strong support for screening and support services regarding previous mental health concerns such as depression and anxiety. Therefore, these results suggest that clinical implications for those mothers with previous mental health history may be a greater need for case management. Given the increased risk of development of PPD cited in previous studies, ${ }^{27-29}$ and the results of the current study indicating that a history of anxiety can negatively impact LOS, case management, and enhancing access to ongoing services may be of particular importance to those mothers with previous mental health concerns.

\section{Implications for mothers without mental health history}

For those mothers without a history of previous mental health concerns, stress related to infants' appearance was among the best predictors of LOS. Previous studies have also cited stress related to the infants appearance as a predictor of PPD. ${ }^{29}$ Complex medical terminology, separation from their infants, and anxiety about long-term consequence of the preterm birth have been suggested to put mothers of preterm infants at higher risk to experience anxiety and depression. ${ }^{12}$ However, for this group with no previous mental health issues, delivery type alone was found to be a significant predictor 
of LOS, while controlling for illness severity. Based on the results of the current study, LOS appears to be impacted by maternal psychological functioning differently for those mothers without previous mental health concerns. Additionally, there has been consistent support in the literature that PPD and psychological distress are important areas to address within this population. ${ }^{19,27-29}$ Therefore, on-site interventions aimed at reducing stress through educational, behavioral, and/or clinical services may be of particular benefit to those mothers without a history of mental health concerns.

\section{Support for universal screening}

The ability to identify these families shortly after birth has significant clinical implications due to the opportunity for them to benefit from early preventative intervention. ${ }^{20}$ Neonates typically stay in the NICU for significant lengths of time, which allows universal screening of mothers of infants while admitted to the NICU as a practical approach to the problem. ${ }^{19}$ Universal screening of all mothers in the NICU can provide numerous benefits, including decreasing the negative stigma, raising awareness of symptoms and their implications, as well as normalizing the experience among mothers of neonates.

Many women are not screened for PPD or anxiety during pregnancy. ${ }^{19}$ Allowing uniform screening of all mothers in the NICU may provide a unique opportunity to identify women with preexisting, undiagnosed, and new-onset symptoms. Regardless of when onset of psychosocial symptoms occurred for the mothers, the risk to infants' health and development is present. Preterm infants are particularly at risk due to the additional risks for growth and developmental problems. ${ }^{19}$ The result of early identification may allow for the development of programs to reduce distress and anxiety in mothers as well as promote optimal development for infants in this high-risk population. ${ }^{20}$

\section{Limitations}

Although the findings for this study offer insight into the impact of maternal psychological functioning on infant LOS in the NICU, there were several limitations. The study resulted in a fairly small participation rate and numerous mothers were unable to be screened. The exclusion criteria of the study, including significant infant congenital anomalies, reading below the eighth grade level, and non-English speaking, led to a large number of mothers with infants hospitalized for greater than 14 days being ineligible. Due to the exclusion of infants with more severe health complications, mothers below an eighth grade reading level, and mothers who were not fluent in English, the generalizability of the findings are limited. Further, a number of those who were eligible declined participation. Thus, the generalizability of this study is further limited, and little is known as to what factors contributed to those who participated and those who did not participate.

An additional limitation was the small sample size. The current sample was collected from a single medical center and therefore cannot be generalized to more ethnically or socioeconomically diverse populations or other types of pediatric settings. Replication is warranted in other sites and clinics, as well as among ethnically diverse populations.

Further limitations to the study were regarding significant correlations among variables. Significant correlations between PPD scores and trait anxiety scores indicate that these constructs are possibly measuring the same or similar phenomena in this population, inclusion of both constructs likely limited the significance of each construct in the overall model. Then finally, due to such a high majority of the mothers screening positive for PPD, there was little variability in the sample, which could also limit the significance of PPD contributing to LOS.

\section{Future research}

Further study and replication is also needed to determine the variance in LOS predicted by maternal psychosocial variables with further statistical modeling. Future studies should investigate a predictive model of LOS for those with previous mental health issues, including variables such as birth weight, prior anxiety, and substance abuse, while controlling for CRIB. It may also be beneficial to possibly stratify the sample by illness severity (CRIB), gestational age, birth weight, etc.

\section{Conclusion}

Despite the need for additional research, there is evidence that maternal psychological functioning is related to NICU LOS. The results of this study also indicate a clinical need to provide routine screening and behavioral health services for mothers in the NICU. The current findings, in addition to future research, will contribute to the development of appropriate interventions targeted at improving parent and child adjustment following a neonate's admission to the NICU, as well as improve the health and quality of life of infants seen in the NICU and the mothers of these neonates.

\section{Acknowledgments}

The authors would like to thank the staff of the Neonatal Intensive Care Unit at the Children's Hospital of Oklahoma, 
Oklahoma City, OK for their support and assistance with this project. This work was supported in part by an interdepartmental grant through the Section of Perinatal Medicine within the Department of Pediatrics at the University of Oklahoma Health Sciences Center.

\section{Disclosure}

The authors declare no conflicts of interest in this work.

\section{References}

1. Eshbaugh EM. Maternal age and depressive symptoms in a low-income sample. J Community Psychol. 2008;36:399-409.

2. Lefkowitz DS, Baxt C, Evans JR. Prevalence and correlates of posttraumatic stress and postpartum depression in parents of infants in the Neonatal Intensive Care Unit (NICU). J Clin Psychol Medical Settings. 2010;17(3):230-237.

3. Oren DA, Wisner KL, Spinelli M, et al. An open trial of morning light therapy for treatment of antepartum depression. Am J Psychiatry. 2002, 159(4):666-669.

4. Vigod SN, Villegas L, Dennis CL, Ross LE. Prevalence and risk factors for postpartum depression among women with preterm and low-birthweight infants: a systematic review. BJOG. 2010;117(5):540-550.

5. Hamilton BE, Martin JA, Sutton PD. Centers for Disease Control and Prevention, National Center for Health Statistics. Births: preliminary data for 2003. Nat Vital Stat Rep. 2004;53(9):1-17.

6. Melnyk BM, Feinstein NF, Alpert-Gillis L, et al. Reducing premature infants' length of stay and improving parents' mental health outcomes with the Creating Opportunities for Parent Empowerment (COPE) neonatal intensive care unit program: a randomized, controlled trial. Pediatrics. 2006;118:e1414-e1427.

7. Cusson RM. Factors influencing language development in preterm infants. J Obstet Gynecol Neonatal Nurs. 2003;32(3):402-409.

8. Melnyk BM, Alpert-Gillis L, Feinstein NF, et al. Improving cognitive development of low-birth-weight premature infants with the COPE program: a pilot study of the benefit of early NICU intervention with mothers. Res Nurs Health. 2001;24(5):373-389.

9. Pinelli J. Effects of family coping and resources on family adjustment and parental stress in the acute phase of the NICU experience J Neonatal Nurs. 2000;19(6):27-37.

10. Carter JD, Mulder RT, Frampton CMA, Darlow BA. Infants admitted to a neonatal intensive care unit: parental psychological status at 9 months. Acta Paediatr. 2007;96(9):1286-1289.

11. Doering LV, Dracup K, Moser D. Comparison of psychosocial adjustment of mothers and fathers of high-risk infants in the Neonatal Intensive Care Unit. J Perinatol. 1999;19(2):132-137.

12. Madu SN, Roos JJ. Depression among mothers with preterm infants and their stress-coping strategies. Soc Behav Pers. 2006;34(7):877-890.

13. Bendetti F, Colombo C, Pontiggia A, Bernasconi A, Florita M, Smeraldi E. Morning light treatment hastens the antidepressant effect of citalopram: a placebo-controlled trial. J Clin Psychiatry. 2003;64(6):648-653.

14. Centers for Disease Control and Prevention (CDC). Prevention CfDCa. Prevalence of self-reported postpartum depressive symptoms. $M M W R$ Morb Mortal Wkly Rep. 2008;57(14):361-366.

15. Gennaro S. Postpartal anxiety and depression in mothers of term and preterm infants. Nurs Res. 1988;37(2):82-85.

16. Logsdon MC, Davis DW, Birkimer JC, Wilkerson SA. Predictors of depression in mothers of preterm infants. J Soc Behav Pers. 1997; 12:73-88.
17. Veddovi M, Kenny DT, Gibson F, Bowen J, Starte D. The relationship between depressive symptoms following premature birth, mothers' coping style, and knowledge of infant development. J Reprod Infant Psychol. 2001;19(4):313-323.

18. McCabe K, Blucker R, Gillaspy JA, et al. Reliability of the postpartum depression screening scale in the neonatal intensive care unit. Nurs Res. 2012;61(6):441-445.

19. Mounts KO. Screening for maternal depression in the neonatal ICU. Clin Perinatol. 2009;36(1):137-152.

20. Zelkowitz P, Bardin C, Papageorgiou A. Anxiety affects the relationship between parents and their very low birth weight infants. Infant Ment Health J. 2007;28(3):296-313.

21. Meyer EC, Garcia Coll CT, Seifer R, Ramos A, Kilis E, Oh W. Psychological distress in mothers of preterm infants. J Dev Behav Pediatr. 1995;16(6):412-417.

22. Singer LT, Fulton S, Davillier M, Koshy D, Salvator A, Bailey JE. Effects of infant risk status and maternal psychological distress on maternal-infant interactions during the first year of life. J Dev Behav Pediatr. 2003;24(4):233-241.

23. Assel M, Landry SH, Swank PR, Steelmand L, Miller-Loncar CC, Smith KE. How do mothers' childbearing histories, stress, and parenting affect children's behavioral outcomes? Child Care Health Dev. 2002; 28(5):359-368.

24. Muller-Nix C, Forcada-Guex M, Pierrehumbert B, Jaunin L, Borghini A, Ansermet F. Prematurity, maternal stress and mother-child interactions. Early Hum Dev. 2004;79(2):145-158.

25. Pierrehumbert B, Nicole A, Muller-Nix C, Forcada-Guex M, Ansermet F. Parental post-traumatic reactions after premature birth: implications for sleeping and eating problems in the infant. Arch Dis Child Fetal Neonatal Ed. 2003;88(5):400F-404F.

26. Landry S, Smith KE, Swank PR. The importance of parenting during early childhood for school-age development. Dev Neuropsychol. 2003;24(2-3):559-591.

27. Rogers CE, Kidokoro H, Wallendorf M, Inder TE. Identifying mothers of very preterm infants at-risk for postpartum depression and anxiety before discharge. J Perinatol. 2013;33(3):171-176.

28. Poehlmann J, Fiese BH. The interaction of maternal and infant vulnerabilities on developing attachment relationships. Dev Psychopathol. 2001;13(1):1-11.

29. Hynan MT, Mounts KO, Vanderbilt DL. Screening of parents of highrisk infants for emotional distress: rationale and recommendations. J Perinatol. 2013:748-753.

30. Beck CT, Gable RK. Postpartum Depression Screening Scale Manual. Los Angeles, CA: Western Psychological Services; 2002.

31. Spielberger CD, Gorsuch RL, Lushene R, Vagg PR, Jacobs GA. Manual for the State-Trait Anxiety Inventory (Palo Alto, CA, Consulting Psychologists Press).

32. Miles MS. Parents of critically ill premature infants: Sources of stress. Critical Care Nursing Quarterly. 1989;12(3):69-74.

33. Logsdon MC. The Postpartum Support Questionnaire: A Measure of Social Support in Postpartum Women. 2nd ed. New York, NY: Springer; 2002.

34. Network TI. The CRIB (clinical risk index for babies) score: a tool for assessing initial neonatal risk and comparing performance of neonatal intensive care units. The Lancet. 1993;342(8865):193-198.

35. Beck CT. Recognizing and screening for postpartum depression in mothers of NICU infants. Adv Neonatal Care. 2003;3(1):37-46. 
International Journal of Women's Health

Dovepress

\section{Publish your work in this journal}

The International Journal of Women's Health is an international, peerreviewed open-access journal publishing original research, reports, editorials, reviews and commentaries on all aspects of women's healthcare including gynecology, obstetrics, and breast cancer. The manuscript management system is completely online and includes Visit http://www.dovepress.com/testimonials.php to read real quotes from published authors.

Submit your manuscript here: http://www.dovepress.com/international-journal-of-womens-health-journal 